\title{
Ensemble Machine Learning Methods for better Dynamic Assessment of Transformer Status
}

\author{
Soham Ghosh ${ }^{1}$ (D) Sreejata Dutta ${ }^{2}$
}

Received: 2 October 2020/Accepted: 15 April 2021/Published online: 19 May 2021

(C) The Institution of Engineers (India) 2021

\begin{abstract}
Analyzing dissolved gases in the transformer's mineral oil helps to detect and classify the systemic faults in electric power transformers. Formerly, empirical methods such as Rogers ratio, Duval triangles 1-4-5, and pentagons 1-2 were used for transformer fault classification. Loose fit for every transformer type is one of the most prominent disadvantages of conventional methods. Formulating robust machine learning algorithms, such as the decision trees, can significantly overcome the loose fit issue. This paper focuses on implementing four different decision tree algorithms, including a regular decision tree classifier, a bagging classifier, a boosting classifier, and a stacking classifier to classify six different transformer fault types distinctly. Further, this study shows that the efficacy and accuracy of the four mentioned classifiers could be far exceeded when combined using a wisdom of the crowd approach. The wisdom of the crowd approach essentially merges the predicted classes from the four individual classifiers and decides on the final prediction via a hardvoting routine. The computational evaluation revealed that the given voting approach could significantly improve power transformers' online diagnostic accuracy up to $91 \%$, thus aiding early forecast of power transformers' preventive maintenance.
\end{abstract}

Soham Ghosh

sghosh27@asu.edu

1 Department of Electrical Engineering, Arizona State University, Tempe, AZ 85281, USA

2 Department of Biostatistics \& Data Science, University of Kansas Medical Center, Kansas City, KS 66160, USA
Keywords Dissolved gas analysis - Ensemble learning · Random forest $\cdot$ XG boost $\cdot$ Stacking classifier . Transformer protection

\section{Introduction}

Power transformers are the single most expensive element of a power substation and form an essential part of an electrical power grid. Transformer problems typically arise either from insulation and winding defects or are induced by operation levels exceeding the device's capability. Thermal and voltage stresses, along with moisture, oxygen, and dissolved contaminants, can further accelerate the insulation deterioration in a power transformer [1]. In case a transformer failure occurs at a substation, a temporary standby transformer is typically deployed, while repairs are being performed in the manufacturer's facility. However, the post-fault transportation and field wiring of standby transformers result in significant downtime and poor system average interruption duration index (SAIDI).

Periodic routine maintenance and the installation of online monitoring systems with an overload detection capability can help to identify early transformer failures. Online monitoring systems can further prevent expensive unscheduled repairs through planned preventive maintenance. During the preventive maintenance outages, the system operator, in most cases, can reconfigure the network to allow uninterrupted service to the end-user.

Dissolved gas analysis of the transformer mineral oil is one of the techniques which is often used in online monitoring systems. Online dissolved gas analysis (DGA) is a mature technology developed in the mid-1990s for transformer fault type identification. The analysis, as proposed in $[2,3]$, uses a membrane extraction technology in 
Table 1 Classification report of the decision tree classifier

\begin{tabular}{llll}
\hline Fault type & Precision & Recall & F1-score \\
\hline $\mathrm{D}_{1}$ & 0.68 & 0.79 & 0.73 \\
$\mathrm{D}_{2}$ & 0.83 & 0.67 & 0.74 \\
$\mathrm{D}_{3}$ & 0.81 & 0.90 & 0.85 \\
$\mathrm{~T}_{1}$ & 0.89 & 0.85 & 0.87 \\
$\mathrm{~T}_{2}$ & 0.60 & 0.75 & 0.67 \\
$\mathrm{~T}_{3}$ & 0.91 & 0.75 & 0.82 \\
Accuracy & & & 0.80 \\
Simulation time without hyperparameter tuning & & \\
Simulation time with hyperparameter tuning & & $\mathrm{s}$ \\
\hline
\end{tabular}

conjunction with Fourier transform infrared (FTIR) spectroscopy to detect key fault gas signatures except for hydrogen. Detailed analysis of these gases in the transformer's mineral oil can help in the early detection of several transformer fault types, including partial discharges (PD), low-energy discharges (LED), high-energy discharges (HED), and thermal faults (TF). The leading gases identified in the DGA monitoring process include saturated and unsaturated hydrocarbons, carbon oxides, and other atmospheric gases: methane $\left(\mathrm{CH}_{4}\right)$; ethane $\left(\mathrm{C}_{2} \mathrm{H}_{6}\right)$; ethylene $\left(\mathrm{C}_{2} \mathrm{H}_{4}\right)$; acetylene $\left(\mathrm{C}_{2} \mathrm{H}_{2}\right)$, carbon monoxide $(\mathrm{CO})$; carbon dioxide $\left(\mathrm{CO}_{2}\right)$, hydrogen $\left(\mathrm{H}_{2}\right)$; oxygen $\left(\mathrm{O}_{2}\right)$; and nitrogen $\left(\mathrm{N}_{2}\right)$.

Several methods $[4,5]$ somewhat correlate the gases produced in mineral oil transformers with certain fault types. Commonly used fault identification methods include Rogers ratio, Duval triangles 1-4-5, and pentagons 1-2, Doernenburg ratios, and Key gas. Though providing a good starting point for general fault detection, these conventional methods are often not tailored to detect and classify individual transformers' fault types. Given that the transformer's mineral oil and insulating medium are unique to every batch (or model), a custom fault classification algorithm can provide more accurate results if trained on the given transformer batch (or model). Another practical problem with the conventional methods in fault classification is their inconsistency. Often, due to their specific empirical formulations, different conventional methods provide varied interpretations.

A more tailored approach to eliminate the loose fitting problem would involve using machine learning algorithms to classify the fault types of a given transformer batch (or model). The trained decision tree-based monitoring system can be deployed with each transformer for online DGA and fault classification. For instance, the well-known C4.5 algorithm demonstrated satisfactory performance in field tests by reducing the training data and improving classification performance by repeatedly training the decision tree while deleting the misclassified instances by the current decision tree $[6,7]$.

The limitations of the conventional methods in terms of low accuracy were extensively documented in [8], with the authors proposing a decision transformer fault diagnostic method (DTFD). A combined framework of fault diagnosis was built while adopting from Rogers' method, the Duval triangle method, and the IEC standard 60599. While an accuracy ranging from $48.5 \%$ to $81.7 \%$ was reported by the authors using conventional methods, the combined DTFD framework yielded $85.2 \%$ diagnostic accuracy with ample room for accuracy improvements.

Another supervised machine learning model used in the transformer's fault diagnosis is support vector machine (SVM). SVM is known for its fast training time and the ability to classify with reasonable accuracy even for a smaller training set. The SVM algorithm provides satisfactory performance only when there is a limited number of classes in the classification problem. As reported in [9], the algorithm could categorize a mutually exclusive multiclass classification with three categories: thermal fault, low-energy discharge, and high-energy discharge. Adding more classes to an SVM algorithm complicates the optimal hyperplanes separation process.

Several other past researches suggested using improved decision tree-based machine learning algorithms over conventional DGA methods [10-13]. However, a clear research gap exists in combining the predictions from a group of ensemble tree-based predictors to improve accuracy. This paper contributes to the research area by building upon existing decision tree-based transformer fault classification research to propose an unique wisdom of the crowd voting approach using ensemble tree-based classifiers. A set of classifiers consisting of a decision tree, random forest (bagging), XG boost (boosting), and stacked classifier (stacking) is used to produce a more robust 
classifier model. Aggregating the predicted class from each classifier with maximum votes show a much superior performance compared to the individual decision tree classifiers. Further, to yield optimum performance, the hyperparameters are tuned for each classifier, and the bestperforming set is selected.

The paper's remaining is organized as follows: a general overview of the data set used is presented, followed by a summary of the importance of an online monitoring system for the dynamic assessment of transformer status. An outline of the decision tree algorithm in conjunction with other computationally robust tree-based ensemble algorithms is illustrated, along with an elaboration on the adapted voting classifier. Finally, the paper expands on how the ensemble machine learning methods are implemented for transformer fault classification, the corresponding results, and a conclusion to identify the future scope of work.

\section{Data Description}

This paper's dataset is obtained from IEEE DataPort ${ }^{\mathrm{TM}}$ [14] and includes the concentrations of gases dissolved in a faulted transformer's mineral oil along with the corresponding fault types. The dataset contains the concentrations of $\mathrm{H}_{2}, \mathrm{CH}_{4}, \mathrm{C}_{2} \mathrm{H}_{6}, \mathrm{C}_{2} \mathrm{H}_{4}$, and $\mathrm{C}_{2} \mathrm{H}_{2}$ in the transformer's mineral oil. The six fault types include arc discharge (D1), partial discharge (D2), spark discharge (D3), low-temperature overheating (T1), medium-temperature overheating (T2), and high-temperature overheating (T3).

As a part of data preprocessing, feature scaling is performed by converting the raw data into ratios. The set of ratios include $\mathrm{R} 1\left(\mathrm{CH}_{4} / \mathrm{H}_{2}\right), \mathrm{R} 2\left(\mathrm{C}_{2} \mathrm{H}_{2} / \mathrm{C}_{2} \mathrm{H}_{4}\right), \mathrm{R} 3\left(\mathrm{C}_{2} \mathrm{H}_{2} /\right.$ $\left.\mathrm{CH}_{4}\right), \mathrm{R} 4\left(\mathrm{C}_{2} \mathrm{H}_{4} / \mathrm{C}_{2} \mathrm{H}_{6}\right)$, and $\mathrm{R} 5\left(\mathrm{CO} / \mathrm{CO}_{2}\right)$. The preprocessing step also include dividing the dataset into a 70:30 train-test split.

Initial analysis and modeling are performed using Python 3.8.8 on a standard windows system with an i5 processor. The models are trained on the training split and evaluated using the test split. Full-scale decision trees are implemented using Python's Scikit-Learn and XG boost libraries, while DESlib is used to implement stacking. Python's GridsearchCV is used for hyperparameter tuning for all four classifiers.

\section{Dynamic Assessment of Transformer Status}

Major equipment like circuit breakers and transformers in high voltage substations has been traditionally monitored using an online condition monitoring system. With the advancements in microprocessor relays, transformer

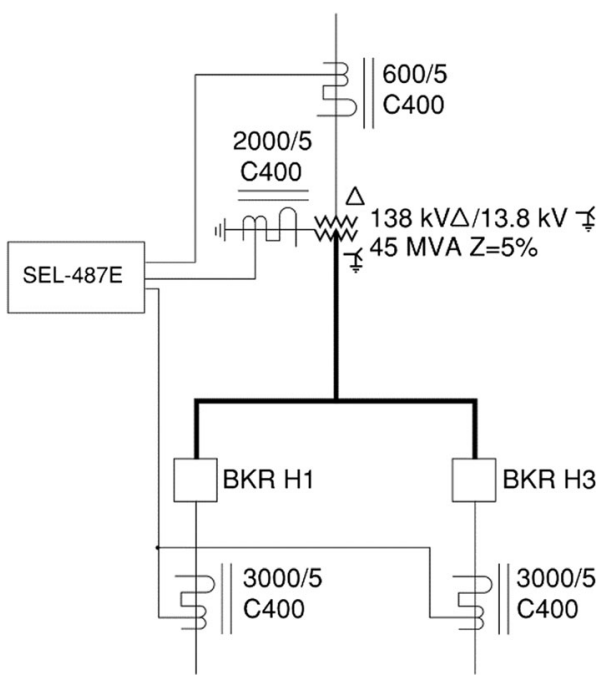

Fig. 1 One relay differential protection scheme with the drawback of unclear fault location

protection relays can detect seemingly benign turn-to-turn faults. For instance, the SEL-487E transformer protection relay can detect faults in as little as two (2) percent of the total electrical wiring. Efficient protection relays can be further combined with innovative DGA monitors, such as Vaisala Optimus ${ }^{\mathrm{TM}}$ OPT100, for in-depth analysis of the transformer health. The benefits of installing a basic transformer dynamic assessment system are many and are described as follows.

\section{One or Two Relay Differential Protection Schemes}

Often, while configuring the current transformer (CT) string for differential protection, the transformer differential protection zone extends beyond the transformer CTs. As shown in Fig. 1, for a dual-breaker transformer terminal, the bold line representing the bus connecting the $13.8 \mathrm{kV}$ low side transformer winding to breakers $\mathrm{H} 1$ and $\mathrm{H} 3$ is included in the transformer's differential zone of protection. With a single (SEL-487E) or dual-primary relay setup (SEL-487E and SEL-387E), the transformer's overall differential element cannot pinpoint if the fault is on the transformer or the bus element. As such, a fault in the bus will trigger a transformer differential fault to trip both the upstream and downstream breakers. Post-fault investigations and root-cause analysis in these cases can be streamlined if the electrical data from the transformer protection relay and dissolved gas data from the DGA monitored are available for comparison. In a typical bus fault scenario, the pre and post-fault dissolved gas data consistency in conjunction with a differential trip can pinpoint the fault location outside the transformer unit.

Moreover, in a parallel two transformer setup, an internal fault can be either confirmed using electrical data 


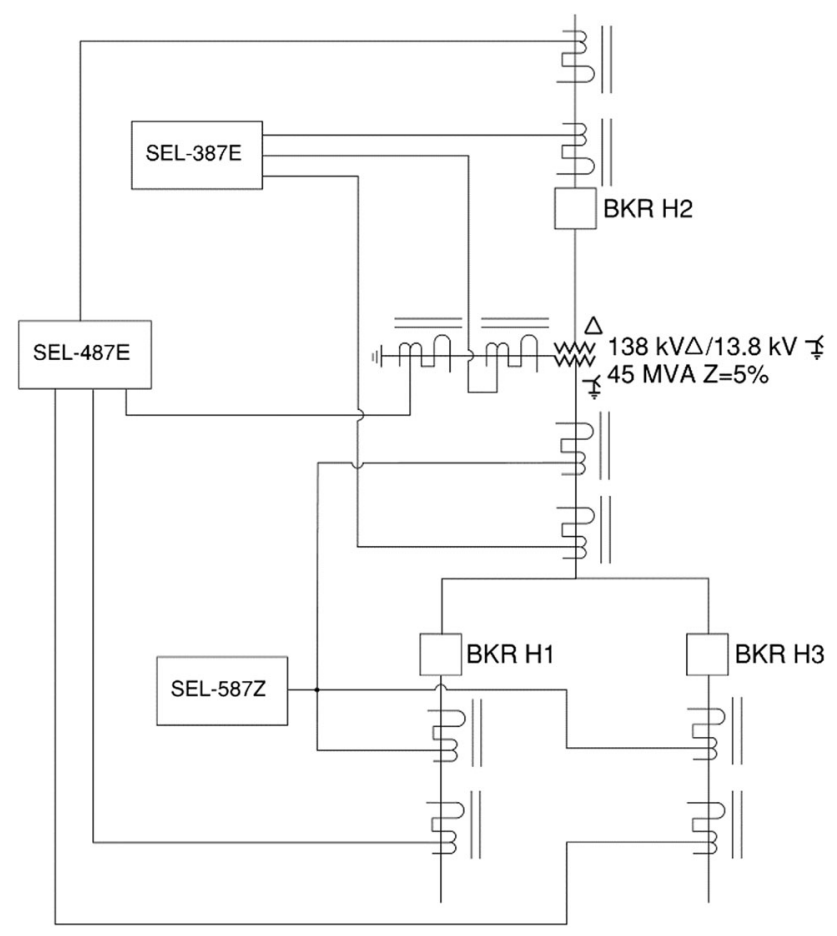

Fig. 2 Three-relay differential protection scheme lacking predictive maintenance insight

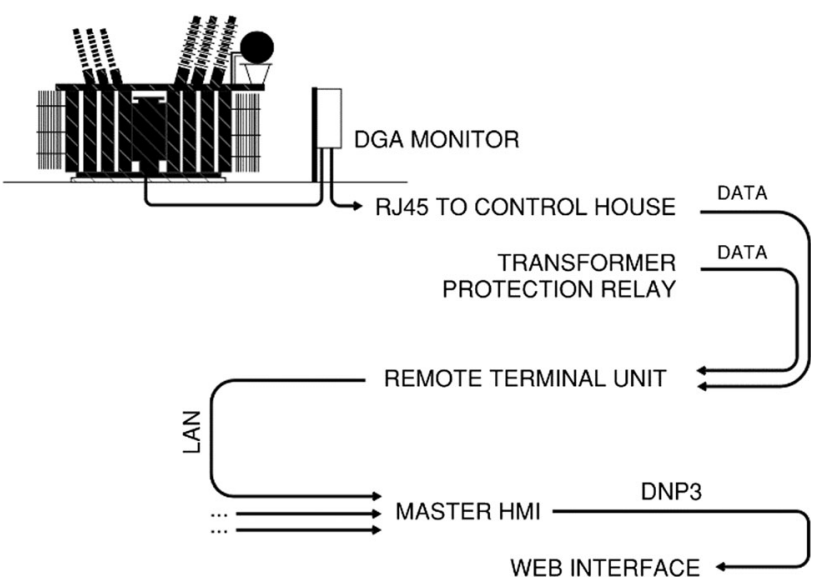

Fig. 3 Transformer electrical and dissolved gas data aggregator

from the transformer protection relay or the dissolved gas data from the DGA monitoring system. In case of an internal fault in one of the two transformers, predictive maintenance can be scheduled on the second transformer to analyze the transformer's health once the first transformer is back online.

\section{Three Relay Differential Protection Scheme}

A more complex arrangement with three relays and additional CT strings can help determine an accurate fault location for a dual-breaker transformer arrangement. As shown in Fig. 2, an overall transformer differential 870 relay (SEL-487E) combined with a transformer differential 87T relay (SEL-387E) and a bus differential 87B relay (SEL-587Z) is used to achieve a three-relay differential protection scheme.

The obvious drawback in the three-relay protection scheme is that it requires an extra set of bushing CTs and the development of three separate relay settings [15]. The other limitation of such a system is that it fails to predict future preventative maintenance issues in the absence of a DGA monitoring system.

When combined with DGA monitors, transformer protection relays can predict possible fault conditions on service transformers with a high degree of accuracy. Figure 3 shows a feasible data collection route using DGA monitors and transformer protection relays. The field data can be aggregated by a remote terminal unit (RTU) to be transmitted to a master human-machine interface (HMI). The aggregated data from electrical relays and DGA monitors can often be analyzed remotely by logging into a secured web interface connected to the master HMI by using the DNP3 protocol.

\section{Decision Trees, Ensemble Learning, and Voting Classifier}

The methods used in this paper comprise of four different machine learning algorithms which can individually detect and classify the probable fault type in a power transformer. The algorithms are traditional decision trees, ensemblebased bagging, ensemble-based boosting, and the stacking classifier.

Further, to improve the overall fault classification, a combination of the four classifiers is used to represent the wisdom of the crowd voting approach. A brief overview of each of the classifiers and their related hyperparameters is discussed as follows:

\section{Decision Tree Classifier}

Decision trees closely mimic human decision-making and divide the data into smaller sub-sections based on specific features' values until all the target variables (or features) fall under one category. Decision trees are known for their robust predictions for both classification and regression problems. The robustness lies in the fact that it requires minimum data preparation in terms of scaling or centering [16].

Classification and regression tree (CART) algorithm is used in this paper. The CART algorithm produces binary trees, unlike other algorithms such as ID3, which can produce more than two children per node. The process 
works by segregating the dataset into two subsets using a single feature $k$ with its corresponding threshold $t_{k}$. The feature-threshold pair $\left(k, t_{k}\right)$ produces the purest subset, and decides the threshold for node split.

Gini impurity, $G_{i}$ is used as a measure of impurity. Improvements in subsequent nodes correlate to the decrease in the value of $G_{i}$, with zero (0) representing the best possible value. Equation (1) shows how $G_{i}$ is calculated from the ratio of $p_{(i, k)}^{2}$ for $k$ training instances in node $i$.

$G_{i}=1-\sum_{k=1}^{n} p_{i, k}^{2}$

The Gini impurity is used in conjunction with the CART cost function to produce the purest subset from the featurethreshold pair. Equation (2) represents the mathematical form of the CART cost function. The CART cost function $C_{\left(k, t_{k}\right)}$ is the sum of the Gini impurity $\left(G_{i}\right)$ of the left and right subset, where $m_{i}$ denotes the impurity in either left or right instance, and $m$ denotes the number of instances in both subsets.

$C_{\left(k, t_{k}\right)}=\frac{1}{m} \sum_{i} m_{i} G_{i}, i=$ left, right

Hyperparameters play a crucial role in decision tree performance. For the CART algorithm, the below set of hyperparameters are considered:

(1) Min_sample_split: The least number of samples to split a given node

(2) Max_depth: The maximum tree depth

(3) Min_sample_leaf: The least number of samples required to be at a leaf node

\section{Random Forest Classifier (Bagging)}

Random forest is a parallel ensemble learning technique. The random forest algorithm applies an add-on bootstrap aggregation, known as bagging, to decision trees. In addition to sampling the training records, the algorithm also samples the features $[17,18]$. The algorithm uses the strongest predictor (or feature) in the top split, producing highly correlated bagged trees. Feature sampling aims at reducing the variance, thus keeping the correlation between bagged trees under control.

The mathematical deduction of the expected value and the variance of a random forest is explained as follows:

Let the number of identically distributed random forest trees be $B$. Therefore, the expected value of a single random forest tree $E\left[\hat{f_{r f}}(x)\right]$ is equal to the average of $B$ random forest trees' expected value, $E\left[\frac{1}{B} \sum_{b=1}^{B} T\left(x ; \Theta_{b}\right)\right]$. As shown in Eq. (3), $T\left(x ; \Theta_{\mathrm{i}}\right)$ is a random forest tree with the characteristics $\Theta_{\mathrm{i}}$ of $i^{\text {th }}$ random forest. The characteristics include the split-variables, the cut-points at each node, and the terminal-node values.

$$
E\left[T\left(x ; \Theta_{\mathrm{i}}\right)\right]=E\left[\hat{f_{r f}}(x)\right]=E\left[\frac{1}{B} \sum_{b=1}^{B} T\left(x ; \Theta_{b}\right)\right], \quad i=1, \ldots, B
$$

As $B$ random forest trees are identically distributed but not necessarily independent, the variance of average $B$ trees can be expressed as Eq. (4). The variable $\sigma^{2}$ represents the variance of each tree $(B)$, with $\rho$ being the positive pairwise correlation between variables. Thus, the product of sample correlation and sample variance limit the random forest estimator's overall variance.

$\operatorname{Var}\left[\hat{r_{r f}}(x)\right]=\rho \sigma^{2}+\frac{1-\rho}{B} \sigma^{2}$

The overall result of a random forest classification problem $\hat{C}_{r f}^{B}(x)$ is given by the majority vote $\hat{C}_{b}(x)_{1}^{B}$, where $\hat{C}_{b}(x)$ is the class prediction of the $b^{\text {th }}$ random forest, and $B$ represents a set of ensemble trees. A more detailed explanation of random forests' working algorithm can be found in $[16,18]$. The hyperparameters for random forest are similar to the decision trees and include min_sample_split, max_depth, and min_sample_leaf.

\section{XG Boost Classifier (Boosting)}

XG (eXtreme Gradient) boost is an extension of the sequential ensemble learning technique. Like the gradient boosting method, XG boosts classifier works by improving (or boosting) the individual tree attributes, which led to the misclassification in the previous tree step. Regularizations are included in machine learning models to avoid overfitting problems. In the XG boost, two parameters, alpha (L1regularization) and lambda (L2-regularization), help to regularize the models. An increase in $L 1$ and $L 2$ regularization parameters penalize complex tree models. The XG boost classifier's strengths include its ability to handle sparse and weighted data [19].

The XG boost model, as proposed in [20], aims at minimization of the cost function, $\zeta(\Theta)$ shown in Eq. (5). The function consists of a convex loss function: $\mathrm{L}(\theta)=$ $l\left(y_{i}, \widehat{y_{i}}\right)$ and a model complexity penalty function: $\Omega(\Theta)=\sum_{k=1}^{K} \Omega\left(f_{k}\right)$.

$\zeta(\Theta)=\sum_{i=1}^{n} l\left(y_{i}, \widehat{y}_{i}\right)+\sum_{k=1}^{K} \Omega\left(f_{k}\right)$

Equation (5) includes functions as parameters and is thus trained in an additive manner. This equation can be 
approximated into a second-order approximation using Taylor's expansion series with the final equation involving a gradient and a Hessian, as shown in Eq. (6).

$$
\begin{aligned}
\zeta(\Theta) & \approx \sum_{i=1}^{n}\left[l\left(y_{i}, \hat{y}^{(t-1)}\right)+f_{t}\left(x_{i}\right)\right]+\Omega\left(f_{t}\right) \\
& =\sum_{i=1}^{n}\left[l\left(y_{i}, \hat{y}^{(t-1)}\right)+g_{i} f_{t}\left(x_{i}\right)+\frac{1}{2} h_{i} f_{t}^{2}\left(x_{i}\right)\right]+\Omega\left(f_{t}\right)
\end{aligned}
$$

where $g_{i}=\partial_{\hat{y}^{(t-1)}} l\left(y_{i}, \hat{y}^{(t-1)}\right)$ and $h_{i}=\partial_{\hat{y}^{(t-1)}}^{2} l\left(y_{i}, \hat{y}^{(t-1)}\right)$. Further, $\Omega\left(f_{t}\right)$ can be, written as:

$\Omega\left(f_{t}\right)=\gamma T+\frac{1}{2} \lambda \sum \omega_{j}^{2}$, where $\gamma$ and $\lambda$ being parameters, $T$ the number of terirhinal leaves, and $\omega$ assigned as the score in each leaf.

Equation (6) can be further tweaked to produce a scoring function that can measure the quality of a tree structure $q$, as noted in Eq. (7). For an excellent mathematical analysis of regularized learning objectives and scoring functions, the readers are encouraged to refer [21].

$\bar{\zeta}^{(t)}(q) \approx-\frac{1}{2} \sum_{j=1}^{T} \frac{\left(\sum_{i \in I_{j}} g_{i}\right)^{2}}{\sum_{i \in I_{j}} h_{i}+\lambda}+\gamma T$

Unlike the previously discussed classifiers, the XG boost has a massive array of hyperparameters. The primary hyperparameters balance overfitting with accuracy and computational complexity. The primary hyperparameters of the $\mathrm{XG}$ boost classifier are:

(1) N_estimators: The number of boosted trees

(2) Colsample_bytree: The ratio of columns (subsample)

(3) Learning rate: $x g b$ 's "eta"

(4) Max_depth: The maximum tree depth

(5) Reg_alpha: L1 regularization term on weights

(6) Reg_lambda: L2 regularization term on weights

(7) Subsample: Subsample ratio of the training instance

\section{Stacked Classifier (Stacking)}

The stacked classifier is another ensemble learning technique to combine multiple heterogeneous weak learners by using a meta-classifier. The typical steps followed by a stacking classifier are:

(1) Segregating the training set into $k$ buckets, employing a $k$-fold cross-validation technique,

(2) A base model trained on ( $k-1)$ buckets of the training information, and estimates are made for the $k^{\text {th }}$ bucket,

(3) Steps 1-2 are iterated for all the $k$ buckets, allowing the base model to be fitted on the entire training set,

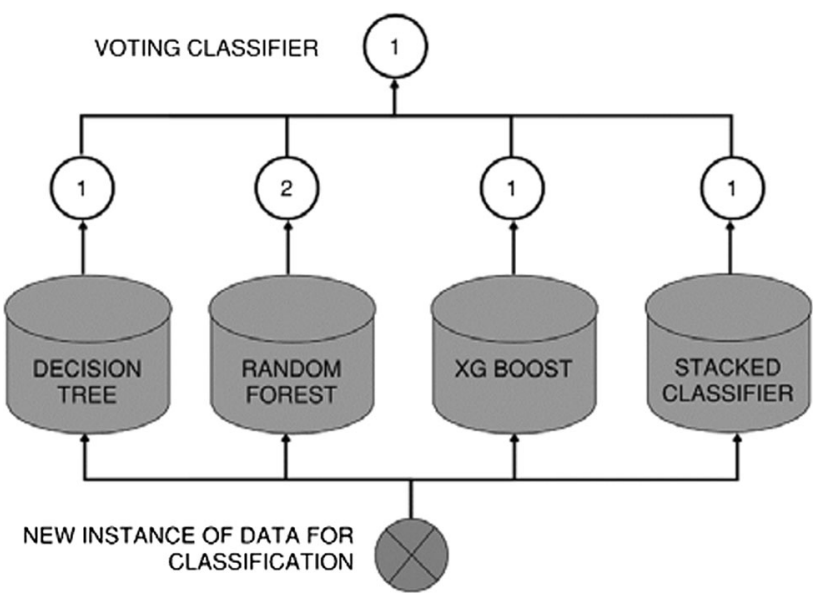

Fig. 4 Combining results from the many classifiers for a voting decision

(4) Steps 1-3 are repeated for other base models (heterogenous learners),

(5) The results from the train set are used as features for the second level meta-classifier,

(6) The second level meta-classifier is to measure performance on the test set.

A pool of classifiers is considered in this study to incorporate the stacking algorithm. The results from this pool of classifiers are then fed into a decision tree metaclassifier for final output. The pool of classifiers includes:

(1) Scalable vector classifier

(2) Gaussian Naive Bayes

(3) K-Neighbors classifier

\section{Wisdom of the Crowd Voting Classifier}

The wisdom of the crowd voting classifier ties all the four classifiers mentioned in the previous sections, to produce a single output. Being a voting mechanism, the wisdom of the crowd classifier does not require separate training. As shown in Fig. 4, a voting classifier considers the result from different classifiers and implements a voting routine. For instance, if three (3) out of four (4) classifiers predict class one (1), the final predicted class from the wisdom of the crowd classifier will also be one (1). Such a voting routine is also known as hard voting. In hard-voting routines, the voting classifier counts the number of each class_instance (predicted class from each of the four classifiers) and declares the result of the test instance (final predicted class) through majority voting. 


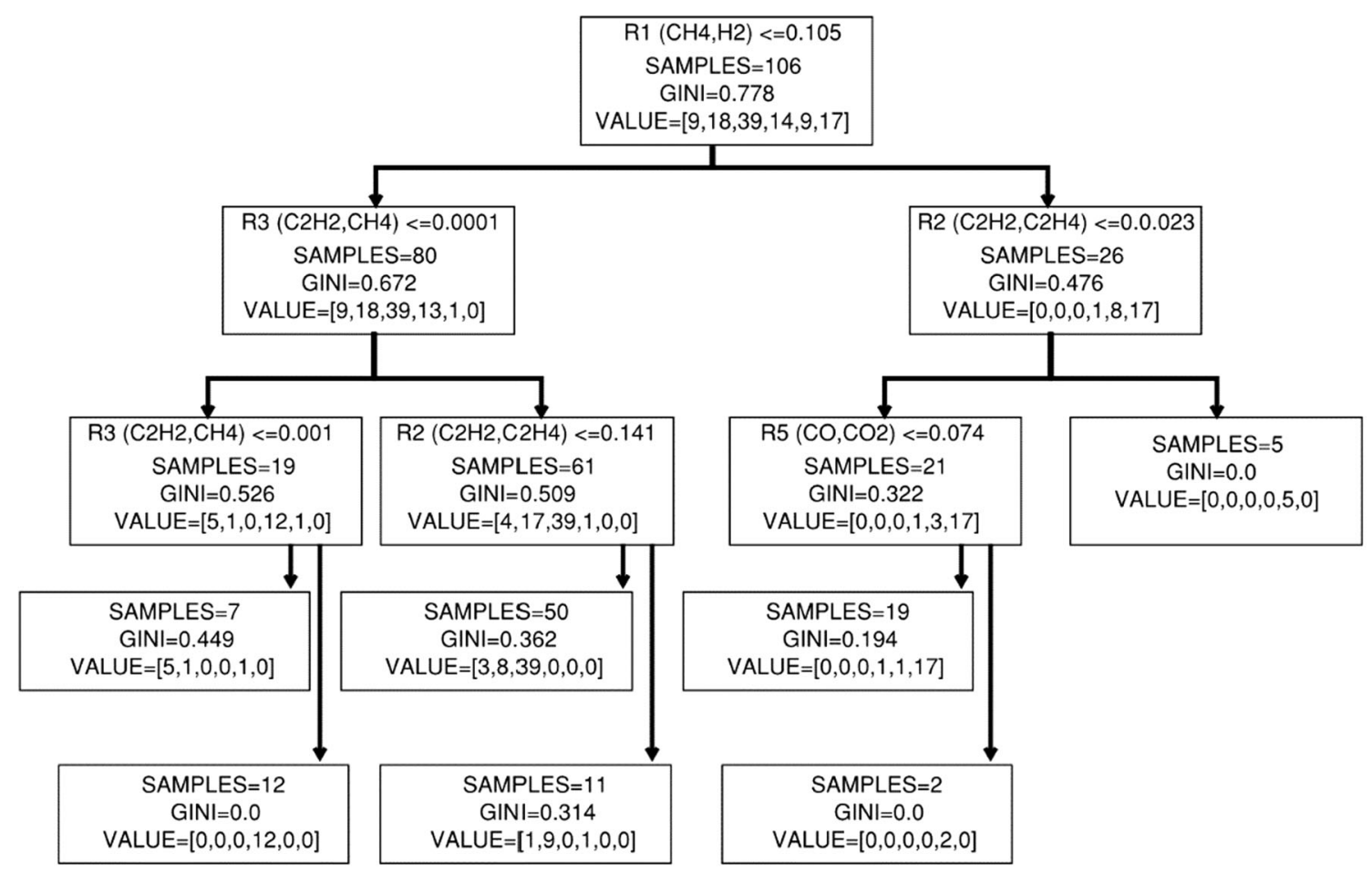

Fig. 5 Flowchart for classification rules of a decision tree model for dissolved gas data

\section{Implementation of Ensemble Machine Learning Methods and Experimental Results}

The preprocessed ratio data are used for training the aforementioned classifiers. A tenfold cross-validation strategy is adapted to train the individual classification models. Hyperparameters are tuned individually for each classification model using GridSearchCV. The set of bestperforming parameter values and the considered array of hyperparameters are elaborated as follows.

\section{Decision Tree Classifier}

Out of the five-set of gas ratios, namely R1, R2, R3, R4, and $\mathrm{R} 5$, the CART algorithm selected the ratio $\mathrm{R} 1$ for splitting the data based on the impurity coefficient, $G_{i}$. Thus, ratio R1 $\left(\mathrm{CH}_{4} / \mathrm{H}_{2}\right)$ forms the decision tree's first node with a threshold value of 0.105 . Figure 5 represents the flowchart generated by the CART classifier trained on the transformer dissolved gas data. Features, along with their corresponding number of samples, Gini impurity, and the array of values are mentioned explicitly in the flowchart. For instance, the Gini impurity of the first node has a sample size of a hundred and six (106), and the values in the first node are $\{9,18,39,14,9,17\}$. Therefore, the Gini impurity can be calculated as:

$$
\begin{aligned}
G_{i}= & 1-\left\{(9 / 106)^{2}+(18 / 106)^{2}+(39 / 106)^{2}+(14 / 106)^{2}\right. \\
& \left.+(9 / 106)^{2}+(17 / 106)^{2}\right\} \approx 0.778
\end{aligned}
$$

For the decision tree classifier, the below set of hyperparameters are considered, while the bestperforming values reported by GridSearchCV are represented in boldface.

'min_samples_split': [3, 5, 10, 20]

'max_depth': [5, 8, 10],

'min_samples_leaf': [1, 2, 3, 4].

Table 1 shows the classification report for the decision tree classifier. A simulation time of $4.2 \mathrm{~s}$ is required to train and tune the decision tree classifier while yielding a test accuracy of $80 \%$.

\section{Random Forest Classifier (Bagging)}

The random forest classifier is evaluated using the same set of hyperparameter values as the decision tree classifier. The GridSearchCV algorithm reported a 'min_sample_split' of $\mathbf{5}$, a 'max_depth' of $\mathbf{8}$, and a 'min_sample leaf' of $\mathbf{1}$ as the best hyperparameter values. Table 2 represents the classification report for the random forest classifier, which exhibited an accuracy of $85 \%$ with a simulation time of $5 \mathrm{~s}$ to train and tune the hyperparameters. 
Table 2 Classification report of the random forest classifier

\begin{tabular}{llll}
\hline Fault type & Precision & Recall & F1-score \\
\hline $\mathrm{D}_{1}$ & 0.85 & 0.89 & 0.87 \\
$\mathrm{D}_{2}$ & 0.87 & 0.72 & 0.79 \\
$\mathrm{D}_{3}$ & 0.84 & 0.97 & 0.90 \\
$\mathrm{~T}_{1}$ & 1.00 & 0.90 & 0.95 \\
$\mathrm{~T}_{2}$ & 0.73 & 0.69 & 0.71 \\
$\mathrm{~T}_{3}$ & 0.85 & 0.82 & 0.84 \\
Accuracy & & 0.85 \\
Simulation time without hyperparameter tuning & $1.8 \mathrm{~s}$ \\
Simulation time with hyperparameter tuning & $5.0 \mathrm{~s}$ \\
\hline
\end{tabular}

Table 3 Classification report of the XG boost classifier

\begin{tabular}{llll}
\hline Fault type & Precision & Recall & F1-score \\
\hline $\mathrm{D}_{1}$ & 0.70 & 0.84 & 0.76 \\
$\mathrm{D}_{2}$ & 0.70 & 0.72 & 0.71 \\
$\mathrm{D}_{3}$ & 0.89 & 0.84 & 0.87 \\
$\mathrm{~T}_{1}$ & 0.90 & 0.90 & 0.90 \\
$\mathrm{~T}_{2}$ & 0.79 & 0.69 & 0.73 \\
$\mathrm{~T}_{3}$ & 0.82 & 0.82 & 0.82 \\
Accuracy & & 0.82 \\
Simulation time without hyperparameter tuning & $2.2 \mathrm{~s}$ \\
Simulation time with hyperparameter tuning & $102.0 \mathrm{~s}$ \\
\hline
\end{tabular}

Table 4 Classification report of the stacked classifier using DESlib

\begin{tabular}{llll}
\hline Fault type & Precision & Recall & F1-score \\
\hline $\mathrm{D}_{1}$ & 0.84 & 0.84 & 0.84 \\
$\mathrm{D}_{2}$ & 0.84 & 0.72 & 0.78 \\
$\mathrm{D}_{3}$ & 0.82 & 0.97 & 0.89 \\
$\mathrm{~T}_{1}$ & 1.00 & 0.90 & 0.95 \\
$\mathrm{~T}_{2}$ & 0.71 & 0.62 & 0.67 \\
$\mathrm{~T}_{3}$ & 0.81 & 0.79 & 0.80 \\
Accuracy & & & 0.84 \\
Simulation time without hyperparameter tuning & $8.5 \mathrm{~s}$ \\
\hline
\end{tabular}

\section{XG Boost Classifier (Boosting)}

For the XG boost classifier, the following set of hyperparameters values are considered, while the best-performing values reported by GridSearchCV are represented in boldface.

'n_estimators': [450, 750, 1050],

'colsample_bytree': [0.55, 0.75, 0.95],

'max_depth': [10, 14, 18, 22],

'reg_alpha': [1.05, 1.25, 1.45],

'reg_lambda': [1.05, 1.25, 1.45],
Table 5 Classification report of the voting classifier

\begin{tabular}{llll}
\hline Fault type & Precision & Recall & F1-score \\
\hline $\mathrm{D}_{1}$ & 0.88 & 0.95 & 0.91 \\
$\mathrm{D}_{2}$ & 0.86 & 0.94 & 0.89 \\
$\mathrm{D}_{3}$ & 0.9 & 0.93 & 0.91 \\
$\mathrm{~T}_{1}$ & 0.98 & 1.00 & 0.98 \\
$\mathrm{~T}_{2}$ & 0.94 & 0.92 & 0.92 \\
$\mathrm{~T}_{3}$ & 0.89 & 0.92 & 0.90 \\
Accuracy & & & 0.91 \\
\hline
\end{tabular}

'subsample': [0.55, 0.75, 0.95].

Table 3 illustrates the XG boost classifier classification report, reporting an accuracy of $82 \%$ with a simulation time of $102 \mathrm{~s}$. The high simulation time for XG boost with hyperparameter tuning is justified given the vast combinatorial set of hyperparameters being tuned.

\section{Stacked Classifier (Stacking)}

DESlib, an open-source implementation [22], was used to implement stacking. No significant improvement in model performance is seen while implementing hyperparameter optimization using grid-search. As such, to reduce model complexity and computation time, the default simulation parameters are regarded. The classification report of the stacked classifier is exhibited in Table 4. The simulation time for the stacked classifier is $8.5 \mathrm{~s}$, with an exhibited accuracy of $84 \%$.

As can be seen from Tables 1, 2, and 3, there is a significant improvement in classification performance using ensemble bagging and boosting methods. However, Table 4 presents an interesting result contrary to the logical expectation of superior performance from a combination of pooled and meta-classifier.

With more factory testing and online monitoring data, the stacked classifier can emerge as a comprehensive training model. Although demonstrating suboptimal performance, it is advised to retain the stacked classifier in the final voting pool and disable it only on a case-by-case basis.

\section{Wisdom of the Crowd Voting Classifier}

As discussed earlier in the paper, the implementation of the voting classifier is likely to exhibit significantly better results in identifying the transformer fault types than any of the aforementioned individual classifiers. The wisdom of the crowd voting classifier is able to predict the transformer fault with $91 \%$ accuracy along with superior precision, 
recall, and F1-scores. Table 5 represents the classification report of the wisdom of the crowd voting classifier.

\section{Conclusion}

Early identification of the transformer at risk can help to prevent catastrophic failures. A multi-pronged analysis using dissolved gas data and electrical data from transformer protection relays can provide clear insight into transformer health. To initiate early warnings for preventive maintenance, accurate classification of the fault is of paramount importance. Through this study, the implementation of the wisdom of the crowd voting classifier emerged as an efficient model in identifying different transformer faults.

As expected, random forests and $\mathrm{XG}$ boost showed better performance in identifying the transformer fault than the decision tree algorithm. However, the performance of the stacked classifier exhibited suboptimal performance. With more factory testing and online monitoring data, the stacked classifier can be trained as a more comprehensive model. Although the overall performance was greatly enhanced by the wisdom of the crowd voting classifier, future studies can further explore ways to enhance the performance of the XG boost and the stacked classifier. One such approach may include using a genetic algorithm, such as particle swarm optimization, for tuning the classifiers' hyperparameters in place of using Python's GridsearchCV.

For brownfield upgrades and retrofits, minor upgrades made to the control enclosure's RTU and associated processing units can help electrical substations support realtime data training and fitting. If in-situ computing power becomes the limiting factor on model scalability, the XG boost algorithm can be used as a stand-alone version, given its effective tree pruning, parallel processing, and inbuilt regularization ability.

Acknowledgements The authors would like to extend their sincere thanks to Christopher P. Martens for his valuable suggestions. The authors would also thank Enwen Li, Linong Wang, and Bin Song for sharing the experimental dissolved gas dataset in IEEE DataPort ${ }^{\mathrm{TM}}$.

\section{References}

1. A. Lux, Online Monitoring of Liquid Immersed Transformers. Electric Power Transformer Engineering, (CRC Press, New York, 2004), p. 3.197

2. D. Chu, H. El Badaly and C. Slemon, Development of an Automated Transformer Oil Monitor. EPRI 2nd Conf. Substation Diagnostics, 1993
3. D. Chu, H. El Badaly and S. C, Status Report on the Automated Transformer Oil Monitor. EPRI 3rd Conference on Substation Diagnostics, 1994

4. IEEE, "C57.104-2019 - IEEE Guide for the Interpretation of Gases Generated in Mineral Oil-Immersed Transformers. IEEE Standards Association, 2019

5. IEC, "60599:2015 - Mineral Oil-Filled Electrical Equipment in Service-Guidance on the Interpretation of Dissolved and Free Gases Analysis. International Standard (IEC), 2015

6. A. Basuki and S. Suwarno, Online Dissolved Gas Analysis of Power Transformers Based on Decision Tree Model. 2018 Conference on Power Engineering and Renewable Energy (ICPERE), pp. 1-6, 2018

7. Z. Zhou, G. Si, J. Chen, K. Zheng and W. Yue, A Novel Method of Transformer Fault Diagnosis Based on K-medios and Decision Tree Algorithm. 1st International Conference on Electrical Materials and Power Equipment, p. 369, 2017

8. S. Ghoneim, D. Mansour, I. Bedir and M. Alharthi, A Decision Transformer Fault Diagnostics System Based on Dissolved Gas Analysis. 2019 21st International Middle East Power Systems Conference (MEPCON), Cairo, Egypt, 2019

9. B. Ramachandran and F. R. Souza, Dissolved Gas Analysis to Identify Faults and Improve Reliability in Transformers Using Support Vector Machines. Clemson University Power Systems Conference (PSC), Clemson, SC, USA, 2016.

10. V. Cheemala, A. N. Asokan and P. Preetha, Transformer Incipient Fault Diagnosis using Machine Learning Classifiers. IEEE 4th International Conference on Condition Assessment Techniques in Electrical Systems (CATCON), 2019.

11. W. Wattakapaiboon and N. Pattanadech, The State of the Art for Dissolved Gas Analysis Based on Interpretation Techniques. International Conference on Condition Monitoring and Diagnosis (CMD), 2016.

12. S. Surawijaya, R. A. Prasojo, W. R. Tamma, I. G. N. Mahendrayana and Suwarno, Diagnosis of Power Transformer Condition using Dissolved Gas Analysis Technique: Case Studies at Geothermal Power Plants in Indonesia. 2nd International Conference on High Voltage Engineering and Power Systems (ICHVEPS), 2019

13. X. Chen, H. Cui and L. Luo, Fault Diagnosis of Transformer Based on Random Forest. Fourth International Conference on Intelligent Computation Technology and Automation, 2011

14. E. Li, L. Wang and B. Song, Dissolved Gas Data in Transformer Oil-Fault Diagnosis of Power Transformers. IEEE DataPort, 2019. https://doi.org/10.21227/h8g0-8z59

15. S. Uddin, A. Bapary, M. Thompson, R. McDaniel and K. Salaunkhe, Application Considerations for Protecting Transformers with Dual Breaker Terminals, 2019.

16. A. Géron, Hands-on Machine Learning with Scikit-Learn, Keras and Tensorflow (O Reilly, Boston, 2020).

17. P. Bruce, A. Bruce, P. Gedeck, Practical Statistics for Data Scientists (O Rilley, Boston, 2020).

18. T. Hastie, R. Tibshirani, J. Friedman, The Elements of Statistical Learning Data Mining, Inference, and Prediction (Springer, London, 2008).

19. A. Gupta, K. Gusain and B. Popli, Verifying the Value and Veracity of Extreme Gradient Boosted Decision Trees on a Variety of Datasets. 11th International Conference on Industrial and Information Systems, pp. 457-462, 2016.

20. J. Brownlee, XGBoost with Python: Gradient Boosted Trees with XGBoost and Scikit-Learn, 2016.

21. T. Chen and C. Guestrin, XG boost: A Scalable Tree Boosting System. Proceedings of the 22nd ACM SIGKDD International Conference on Knowledge Discovery and Data Mining, 2016. 
22. R. M. O. Cruz, L. G. Hafemann and R. Sabour, DESlib: A Dynamic Ensemble Selection Library in Python. arXiv preprint (2018)
Publisher's Note Springer Nature remains neutral with regard to jurisdictional claims in published maps and institutional affiliations. 